\title{
Neurofibromatosis Type 1 in Two Siblings in a Family with Different Presentations - A Series of Two Cases
}

\author{
Parul Dutta ${ }^{1}$, Zohaib Hussain ${ }^{2}$ \\ ${ }^{1}$ Department of Radiology, Gauhati Medical College and Hospital, Guwahati, Assam, India. \\ ${ }^{2}$ Department of Radiology, Gauhati Medical College and Hospital, Guwahati, Assam, India.
}

\section{INTRODUCTION}

"Neurofibromatosis type 1" (NF1) was identified in 1882 by Friedrich Daniel von Recklinghausen, who proposed the term neurofibroma for soft tissue tumors of neural origin found in this syndrome and neurofibromatosis when there are numerous neurofibromas. Neurofibromatosis type 1 (NF1), now also identified as "von Recklinghausen's disease", is a neurocutaneous multiple system disease (phakomatosis). It has an autosomal dominant pattern of inheritance with the NF 1 gene localized on chromosome 17. Owing to this pattern of inheritance, multiple individuals in a single family can be affected. Here, two cases of neurofibromatosis type 1 are presented who happen to be siblings. Evaluation of the elder male sibling led to further investigations and evaluation of the younger female sibling with multiple yet varied characteristic features seen in both.

Case 1 presented with a large extensive plexiform neurofibroma and multiple smaller cutaneous neurofibromas with café-au-lait spots. Case 2 revealed multiple neurofibromas with one of them in left knee showing aggressive infiltration possibly early malignant characteristics along with multiple neuro-parenchymal abnormalities such as low-grade glioma and focal areas of signal intensity (FASI).

\section{PRESENTATION OF CASE}

\section{CASE 1}

25 years old male individual, a daily laborer by occupation presented to the Surgery OPD of this institute.

\section{Chief Complaints}

An extensive pedunculated swelling over the anterior and right lateral aspect of the neck and upper chest wall. The lesion was of insidious onset gradually growing over many years and has extensive overhanging margins reaching up to the umbilicus inferiorly and right axilla laterally. The patient however did not complain of any pain or tenderness of the swelling. No significant past history of injuries, surgeries or any complication of the swelling was given.
Corresponding Author: Dr. Zohaib Hussain H-21 Red Cross Hospital Road, Chandmari, Guwahati-781003, Assam, India.

E-mail: bond007.zohaib@gmail.com

DOI: $10.14260 / j e m d s / 2020 / 429$

How to Cite This Article:

Dutta P, Hussain Z. Neurofibromatosis type 1 in two siblings in a family with different presentations- a series of two cases. J. Evolution Med. Dent. Sci. 2020;9(27):19701973, DOI: $10.14260 /$ jemds/2020/429

Submission 17-04-2020,

Peer Review 03-06-2020,

Acceptance 10-06-2020,

Published 06-07-2020.

Copyright (c) 2020 JEMDS. This is an open access article distributed under Creative Commons Attribution License [Attribution 4.0 International (CC BY 4.0)] 


\section{Family History}

Revealed complaints of multiple swellings in the patient's only sibling, a younger sister. No other significant family history was obtained.

\section{On Examination}

The large lesion is non-tender, soft and firm on palpation. No abnormal bruit heard in auscultation. Multiple $(>10)$ hyperpigmented patches (café-au-lait), largest measuring up to more than $20 \mathrm{~mm}$ along with Multiple smaller nodular soft firm lesions were also noted scattered through out the thoracic and abdominal walls. (Figure 1)

\section{Radiological Evaluation}

On ultrasonography, the lesion appeared solid and heteroechoic with minimal internal vascularity as detected on color doppler interrogation. (Figure 2)

On magnetic resonance imaging, the lesion appears isointense to muscle on T1WI and hyperintense on T2WI. On DWI, the lesion shows patchy diffusion restriction and homogeneous contrast enhancement on post contrast T1WI. The lesion is noted to be confined to the superficial tissue layer without obvious deep muscular infiltration. Multiple smaller similar nodular subcutaneous lesions are also noted scattered throughout the visualized thorax and abdomen. (Figure 3)

\section{Differential Diagnosis}

A provisional diagnosis of a large pedunculated plexiform neurofibroma with multiple smaller neurofibromas was made based on the clinical history, examination coupled with radiological findings, possibly neurofibromatosis type 1 . (caféau-lait spots, a large plexiform and multiple smaller neurofibromas and first degree relative with NF1) On histopathological examination, the large overhanging lesion was revealed to be a neurofibroma.

\section{CASE 2}

Detailed history of case 1 revealed a female sibling, 16 years of age, student by occupation who has complaints of small swelling over the occipital region of scalp on the left side and over the lateral aspect of left knee which were insidious onset and slowly growing in size. Due to lack of obvious symptoms, the patient had consulted a local physician and was managed conservatively so far.

\section{On Examination}

The lesions over scalp and left knee are non-tender, soft and firm on palpation. No abnormal bruit heard in auscultation. No evidence of any other swellings or pigmented skin lesions was found.

\section{Radiological Evaluation}

On ultrasonography, the lesions appeared solid hetero-echoic with minimal internal vascularity as detected on color doppler interrogation. (Figure 4)

On MRI, the lesions over the scalp and left knee appear isointense to muscle on T1WI and hyperintense on T2WI. On
DWI, the lesions show patchy diffusion restriction and homogeneous contrast enhancement on post contrast T1WI. The lesion over the scalp is noted to be confined to the superficial layers without obvious inter or intra muscular infiltration. However, the lesion in the left knee is noted to infiltrate into the underlying vastus, biceps femoris muscles and also in to the lateral aspect of the left knee joint space, suggesting a more aggressive infiltrative pathology. (Figure 5)

MRI of the brain also revealed a T2 and FLAIR hyperintense lesion in the left high frontal lobe without any diffusion restriction or post contrast enhancement, possibly a low-grade glioma. Multiple focal non-enhancing T2/FLAIR hyperintense foci were also noted in the deep periventricular white matter and capsuloganglionic region on the right side, suggestive of focal areas of signal intensity (FASI). (Figure 6) A provisional diagnosis of multiple cutaneous neurofibromas was made based on the clinical history coupled with other (intracerebral) radiological findings, possibly neurofibromatosis type 1. (multiple neurofibromas, a lowgrade glioma, FASI and a first degree relative with NF1) On Histopathological Examination, the lesion of the left knee was revealed to be a neurofibroma with cellular atypia, possibly early malignant changes. (Figure 7)

\section{DISCUSSION}

The defining feature in NF1 is the presence of neurofibromas, peripheral nerve sheath lesions, that are the predominant mode of clinical presentation and disfigurement with NF1.[1] The 'plexiform neurofibroma' is used to characterize a web-like tumor growth affecting multiple nerve fascicles which results in a dense cluster of thickened nerves engulfed by proteinaceous matrix.[2] NF1 is caused by an NF1 gene modification, and the frequency is around 1:4,000 live births. This gene located on chromosome 17 (17q11.2) is a tumor suppressor gene found in its long arm. The lack of function of this gene due to a mutation dictates a rise in cell growth and tumorigenesis. [3]

\section{Criteria for the Diagnosis of Neurofibromatosis Type 1 (as per the National Institutes of Health, 1990 Criteria) $^{[4]}$}

1. 6 or more café au lait spots which are $>5 \mathrm{~mm}$ in the prepubertal group or $>15 \mathrm{~mm}$ in post pubertal group.

2. 2 or more neurofibromas of any type or a single plexiform neurofibroma.

3. Freckling in axilla and/or inguinal regions.

4. Two or more "Lisch nodules" (pigmented iris hamartomas).

5. A typical bony defect, such as dysplasia of the sphenoid bone or cortical thinning of long bones, with or without pseudoarthrosis.

6. One first-degree relative (parent, sibling, son or daughter) who meets above criteria.

Two or more of the above criteria are needed for diagnosis of NF1. ${ }^{[4]} \mathrm{A}$ common occurrence in NF1 are pigmented lesions. Typically, these lesions occur in the early years of life, or can be seen at birth, either as spots (café au lait) or axillary or inguinal freckling.[5] Café au lait spots are deeply 
pigmented maculae and may differ in color from light to dark brown, and may have smooth or uneven borders. Sometimes, there are inguinal and axillary freckles (Crowe's sign) seen. Often common in NF1 are numerous skin neurofibromas, as well as angiomas. Two major clinical types of neurofibromas exist: Localized and Plexiform. The most widespread in NF1 are localized neurofibromas. This grows as a focal mass around the peripheral nerve, with well-defined boundaries. Skin is the commonly affected organ. Frequently affected locations in head and neck are scalp, cheek, and oral cavity.[3,5]

Bony affliction in NF1 such as sphenoid dysplasia and pseudoarthrosis of long bones may be attributed to both external bone loss and osteolytic internal defects. Neurofibromas will rarely be predominantly intraosseous; they typically occur as unilocular, well-defined radiolucency on plain radiography. ${ }^{[3,5]}$

One would also encounter iris hamartomas, VIII nerve neuromas, central nervous system tumors (glioma, glioblastoma), macrocephalus and mental retardation (in up to 40 percent of cases). [3,5]

Most neurofibromas display low attenuation on CT scans, while some may demonstrate density of soft tissue attenuation. ${ }^{[3]}$ Lower density lesions represent a variable number of Schwann cells, and have higher quantities of lipids with cystic changes, and xanthomatous features.[6] Areas with high density are considered to represent regions rich in collagen or cellular density. On magnetic resonance imaging (MRI), lesions display a low signal on T1WI and a high signal on T2WI, with the variable post contrast enhancement. Typical characteristic of neurofibromas is a high peripheral signal with a small area of low central signal in T2 weighted images (bull's eye sign). A related sign can be found on CT; a central area of high attenuation is present in this scenario. ${ }^{[3]}$

On histology, neurofibromas consist of a combination of Schwann cells, perineural cells with endo-neural fibroblasts, and are unencapsulated.[6]

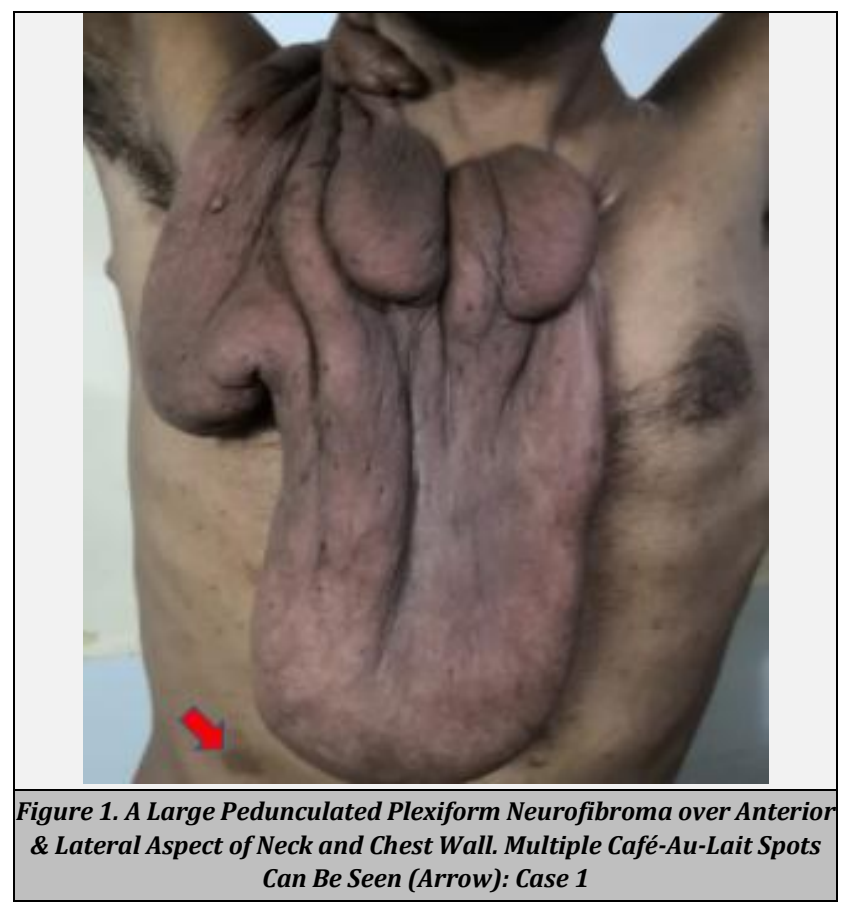

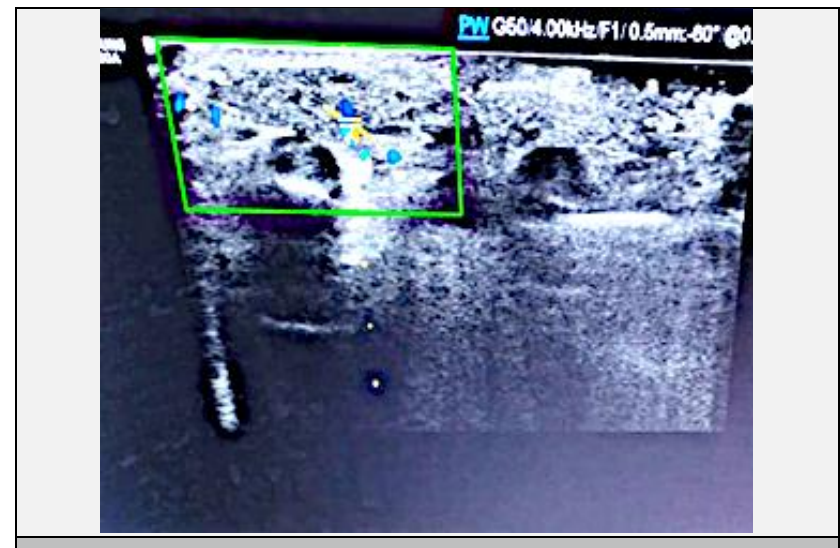

Figure 2. Ultrasonography Findings of the Plexiform Neurofibroma in Case 1 Showing Heteroechogenicity with Minimal Internal Vascularity on Color Doppler Study
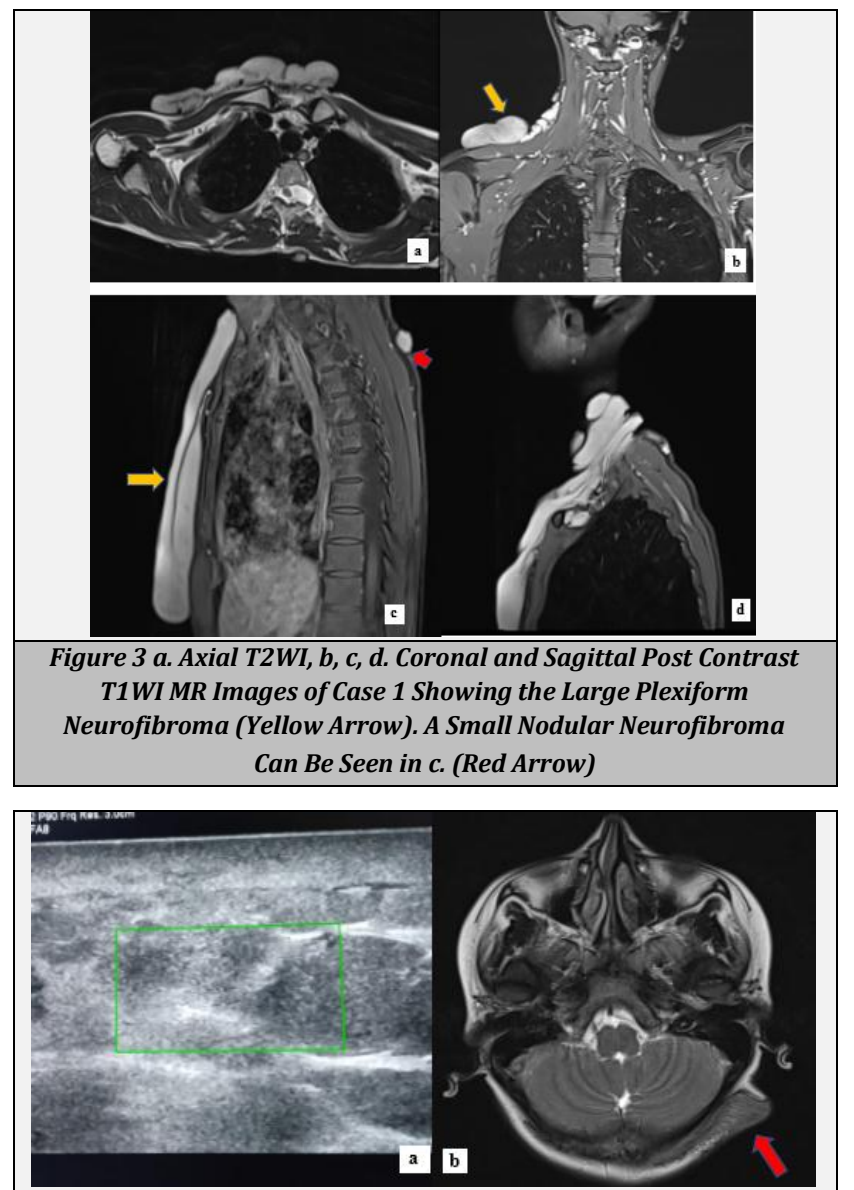

Figure 4a. Ultrasonography, b. Axial T2W MR Images Show Characteristics of the Lesion (Neurofibroma) of the Scalp of Case 2 (Arrow)

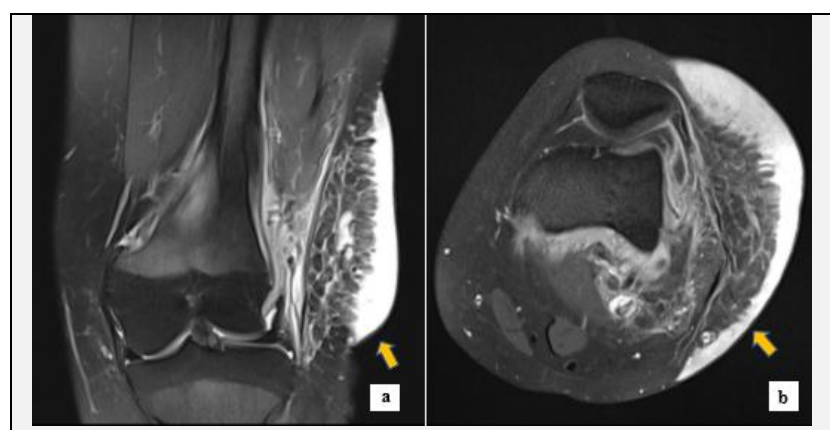

Figure 5a. Coronal T2W and b. Axial Post Contrast T1W MR Images Showing the Lesion (Aggressive Early Malignant Neurofibroma) along the Lateral Aspect of Left Knee of Case 2 (Arrows) 

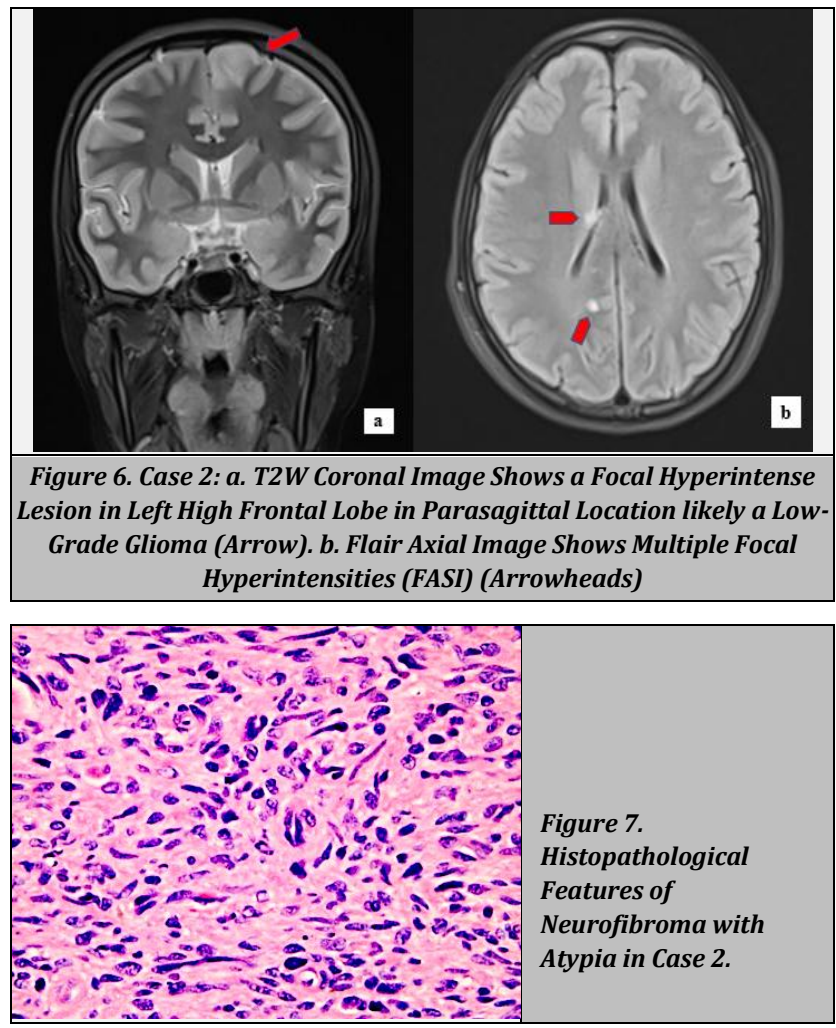

\section{FINAL DIAGNOSIS}

In both the cases presented, the above-mentioned criteria of multiple neurofibromas, a giant plexiform neurofibroma in case 1 , intra cerebral parenchymal abnormalities in case 2, café-au-lait spots in case 1 and first-degree relatives coupled with the biopsy confirmation helped in arriving at a presurgical diagnosis of both of these cases as neurofibromatosis type 1 in two siblings. This helped to further plan management strategies in both siblings separately.

\section{DISCUSSION OF MANAGEMENT}

Total or partial surgical removal of lesions of neurofibromatosis is the remedy of choice to address cosmetic and/or functional issues; it is better to wait for surgery before growth is complete and thus decrease the chance of reoccurrence. Thus, in these cases, it would be advisable to surgically resect the larger plexiform and cutaneous neurofibromas and closely monitor the neurological status in "case 2" with regular annual imaging follow up if asymptomatic. Whenever practicable, complete resection with $1 \mathrm{~cm}$ margins is the treatment of choice for both visible and small tumours. Friedrich et al. proposed that early surgical treatment in children with small-sized plexiform neurofibromas may be helpful, especially in the means of avoiding their progression. Treatment with radiation therapy or chemotherapy is not prescribed. Neurofibroma's malignant transition frequency in NF1 syndrome is 3 to 5 percent. [7]

Such patients with NF1 need to be given genetic counselling because this is an autosomal dominantly transmitted disorder and the risk of transmission to offspring of both sexes is 50 per cent. [4]

\section{CONCLUSIONS}

Neurofibromatosis type 1 is more commonly diagnosed now than previously. The above two cases highlight importance of clinical examination, radiological evaluation, and detailed history taking in diagnosing a case of neurofibromatosis type 1 with reasonable confidence prior to histopathological or surgical biopsy. It also highlights the importance of surveillance in family members of a case of NF1 and also of genetic counselling as autosomal dominant transmission is a feature of this disorder. These cases depict the wide variety of features that may be encountered in a case of NF1 and the fact that all the features of NF1 may not be evident in a single case.

The authors would like to acknowledge the support and help received from the faculties, students, and technical staff, of the Department of Radiology, Gauhati Medical College and Hospital, Guwahati, Assam, India.

\section{REFERENCES}

[1] Korf BR. Plexiform neurofibromas. Am J Med Genet 1999;89(1):31-7.

[2] Jacquemin C, Bosley TM, Svedberg H. Orbit deformities in craniofacial neurofibromatosis type 1 . Am J Neuroradiol 2003;24(8):1678-82.

[3] Hillier JC, Moskovic E. The soft tissue manifestations of neurofibromatosis type 1. Clin Radiol 2005;60(9):960-7.

[4] Aoki S, Barkovich AJ, Nishimura K, et al. Neurofibromatosis types 1 and 2: cranial MR findings. Radiology 1989;172(2):527-34.

[5] Williams VC, Lucas J, Babcock MA, et al Neurofibromatosis type 1 revisited. Pediatrics 2009;123(1):124-33.

[6] Ide F, Shimoyama $T$, Horie $N$, et al. Comparative ultrastructural and immunohistochemical study of perineurioma and neurofibroma of the oral mucosa. Oral Oncol 2004;40(9):948-53.

[7] Friedrich RE, Schmelzle R, Hartman M, et al. Resection of small plexiform neurofibromas in neurofibromatosis type 1 children. World J Surg Oncol 2005;3(1):6. 